\title{
Laparoscopic Resection of Gangrenous Small bowel with acute small bowel obstruction - World's First reported case
}

\author{
Shariful Islam ${ }^{\star, \dagger, 1}$, Otis Payne ${ }^{2}$, Vinoo Bheem ${ }^{3}$, Patrick Harnarayan ${ }^{4}$, Dilip Dan ${ }^{5}$ \\ ${ }^{1}$ MBBS, DM, San Fernando Teaching Hospital, San Fernando, Trinidad and Tobago, Department of Clinical Surgical Science, \\ University of the West Indies, St. Augustine, Trinidad and Tobago \\ ${ }^{2}$ MBBS, San Fernando Teaching Hospital, San Fernando, Trinidad and Tobago, Department of Clinical Surgical Science, University \\ of the West Indies, St. Augustine, Trinidad and Tobago \\ ${ }^{3}$ FRCS, San Fernando Teaching Hospital, San Fernando, Trinidad and Tobago \\ ${ }_{4}^{4}$ FRCS, FACS, San Fernando Teaching Hospital, San Fernando, Trinidad and Tobago, Department of Clinical Surgical Science, \\ University of the West Indies, St. Augustine, Trinidad and Tobago \\ ${ }^{5}$ FACS, San Fernando Teaching Hospital, San Fernando, Trinidad and Tobago, Department of Clinical Surgical Science, University \\ of the West Indies, St. Augustine, Trinidad and Tobago
}

DOI: https://doi.org/10.15520/ijmhs.v9i3.2493

Accepted 9 Mar 2019; Received 1 Feb 2019; Publish Online 28 Mar 2019

Reviewed By: Dr.

Daniel V.

Department: Medical

\begin{abstract}
Introduction: Diagnostic laparoscopy is now frequently used for small bowel obstruction with a varying degree of success. However, emergency laparoscopic resection of gangrenous small bowel has not yet been reported in the English literature. We report the world first case of successful laparoscopic assisted resection of gangrenous small bowel in a 64 year old female with an excellent postoperative outcome.

Presentation of Case: A 64 years old woman with a virgin abdomen presented with a clinical and radiological features of small bowel obstruction. The patient was hemodynamically stable with mild central abdominal distension and tenderness but there was no guarding or rebound tenderness. On diagnostic laparoscopy a loop of gangrenous small bowel was noted secondary to an adhesion band. The patient had a successful laparoscopic assisted resection and extra-corporal anastomosis of the small bowel with an excellent postoperative outcome.

Discussion: Diagnostic laparoscopy plays a vital role for the diagnosis and treatment of selected cases of small bowel obstruction in a virgin abdomen. However, appropriate patient selection as well as the availability of a skilled laparoscopic surgeon is off paramount importance for a successful outcome. Contrast enhanced CT scan is also a useful tool for appropriate patient selection. The morbidity of laparotomy can often be avoided in presence of a skilled laparoscopic surgeon.

Conclusion: Laparoscopic approach can be attempted in selected patients with first episode of SBO and/or anticipated single band obstruction. Similarly, gangrenous small bowel can be resected laparoscopically in highly selected patients in the presence of an experienced laparoscopic surgeon.
\end{abstract}

Key words: Laparoscopy-Resection of gangrenous small bowel-Small bowel obstruction

\section{INTRODUCTION}

Laparoscopic surgery has been established as a preferred method of surgery in almost every field of elective surgery. The unique benefits of this minimally invasive technique in

\footnotetext{
* Corresponding author.

$\dagger$ Email: sss1201198@yahoo.com.
}

both peri and postoperative period are now well recognized by the surgeons as well as by the patients. Many complex procedures are now performed laparoscopically than ever before. Emergency surgeries are also not left behind. Its role in emergency surgery is also rapidly gaining momentum amongst many general and laparoscopic surgeons. Although surgeons have been using laparoscopy for adhesive small bowel obstruction for quite some time, its exact role 


\section{Laparoscopic Resection of Gangrenous Small bowel with acute small bowel obstruction - World's First reported case

in this management is yet to be established. Different case series have documented various degrees of success. However, in all of these series immediate open conversions were performed, once gangrenous bowel was found on diagnostic laparoscopy. To the best of our knowledge, emergency laparoscopic resection of gangrenous small bowel for intestinal obstruction has not yet been reported in the English literature. Open conversion still remains the standard approach. We here by reporting the world's first case of successful laparoscopic resection of gangrenous small bowel in a patient presented with acute small bowel obstruction.

\section{THE CASE:}

A 64 year old woman presented to our accident and emergency department with one day history of central abdominal pain and vomiting. The patient reported that her condition had worsened over the last 6 hours and had not passed any flatus for the last $24 \mathrm{hrs}$. The patient gave no history of change of bowel habit $(\mathrm{COBH})$ or any other constitutional symptoms suggestive of colorectal tumors. She was hypertensive and had no previous abdominal surgery.

On examination in the Accident and Emergency department, the patient was comfortable and her mucous membranes were pink but dry. She was normotensive with a pulse of 84 beats per minute and her SPO2 was $98 \%$ on room air. Abdominal examination revealed mild central abdominal distension with moderate tenderness in the epigastric region on palpation but there was no guarding or rebound tenderness. There was no palpable mass or hernia noted. Digital rectal examination was normal.

\section{Investigations}

Blood investigations revealed an elevated WBC with a normal $\mathrm{Hb}$ and a normal renal function test.

Plain abdominal radiographs demonstrated dilated loops of small bowel.

A CT scan of abdomen and pelvis with intravenous contrast revealed small bowel obstruction with dilated proximal and collapsed distal small bowel. The CT scan also showed moderate free fluid in the abdomen but there was no radiological evidence of any ischemic bowel or any free air (Figures 1 and 2).

\section{Differential Diagnosis}

Small bowel obstruction secondary to bands, internal herniation, tumour or volvulus

\section{Treatment}

After adequate resuscitation the patient was consented for emergency laparoscopy. Under General anesthesia with the patient in supine position, the abdomen was clean and draped. Pneumo-peritoneum was created up to $12 \mathrm{~mm}$ hg with open Hasson technique via a supra-umbilical incision.

At laparoscopy a loop of gangrenous proximal ileum was noted with dilated proximal jejunum and collapsed distal ileum (Figure 3). There was moderate amount of blood stained free fluid in the abdomen and pelvis.

Three other ports one $12 \mathrm{~mm}$ port in supra-pubic area, one $5 \mathrm{~mm}$ port in left lower quadrant and one in right flank

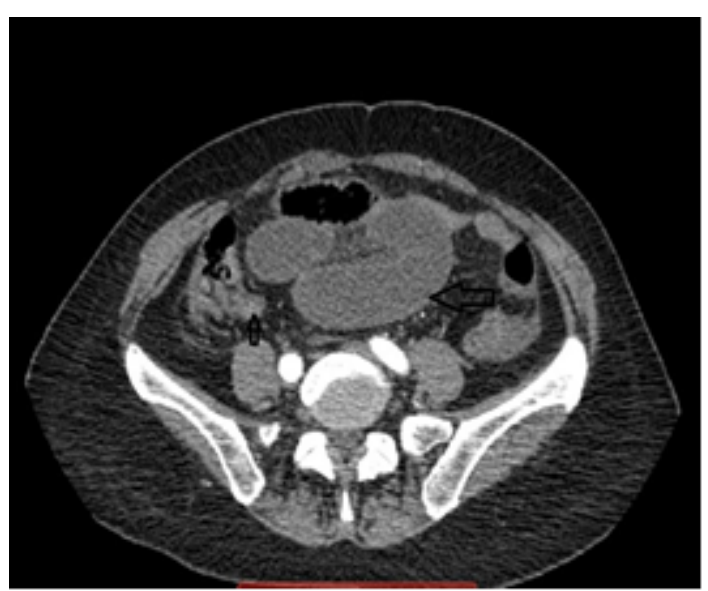

Figure 1. CT scan of abdomen and pelvis showing dilated proximal small bowel (broad arrow)

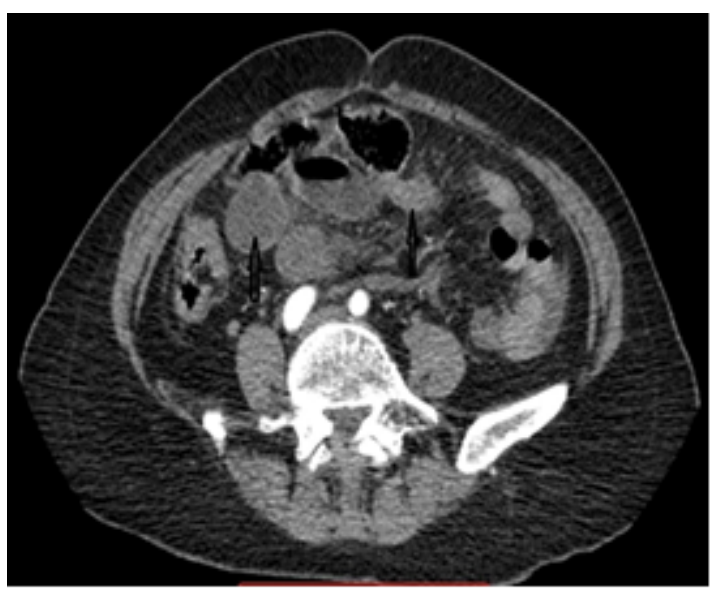

Figure 2. distal collapse small bowel (narrow arrow)

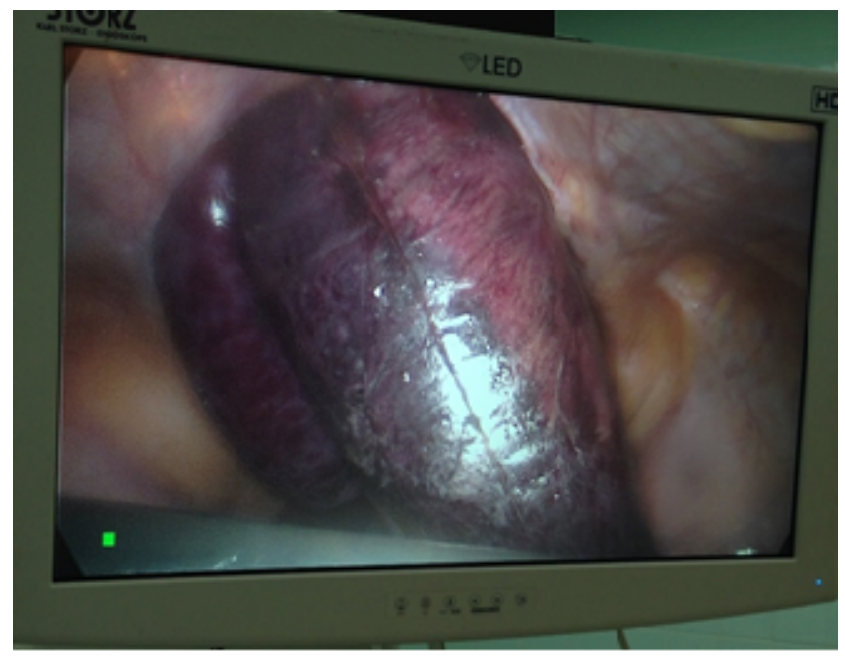

Figure 3. The gangrenous loopof small bowel on initial diagnostic laparoscopy 
were inserted under direct vision. Free fluid was aspirated and the small bowel was followed carefully in a retrograde direction from the ileo-cecal junction serially towards the point of obstruction. A single constricting band was noted from the mid transverse colon to around the loop of proximal jejunum. The adhesive band causing the compression and ischemic necrosis of the segment of ileum was first released and wait for sometimes. However, the bowel failed to return back to its normal colour and there was no visible peristalsis (Figure 4).

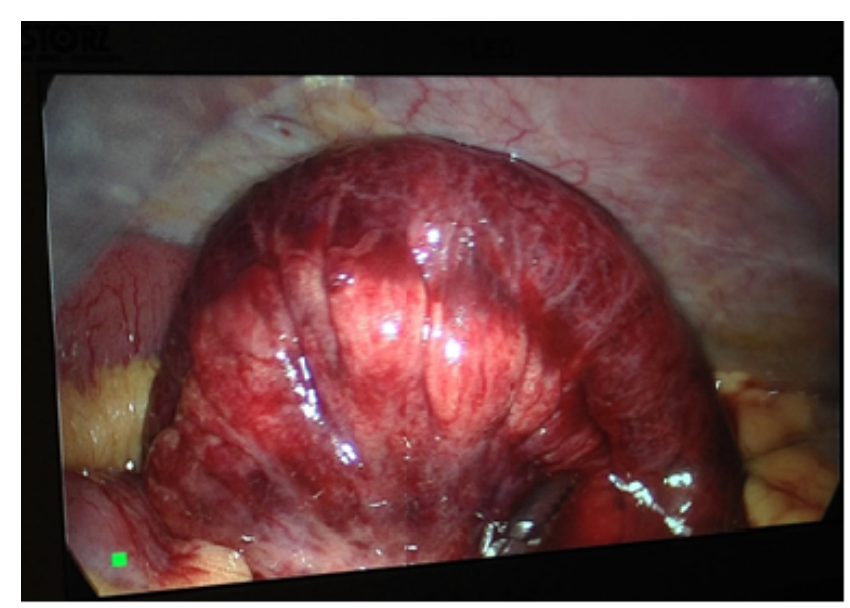

Figure 4. Laparoscopic image of smallbowel after the release of the adhesive band with further observation

Decision was then made to proceed with laparoscopic resection and anastomosis. Approximately 30-40 cm of necrotic small bowel was resected with $45 \mathrm{~mm}$ endo GI stapler and mesenteric blood vessels were taken down carefully with a harmonic scalpel. The necrotic bowel was then removed within a retrieval bag via a supra-pubic incision at the supra-pubic port site (Figures 5 and 6 ).

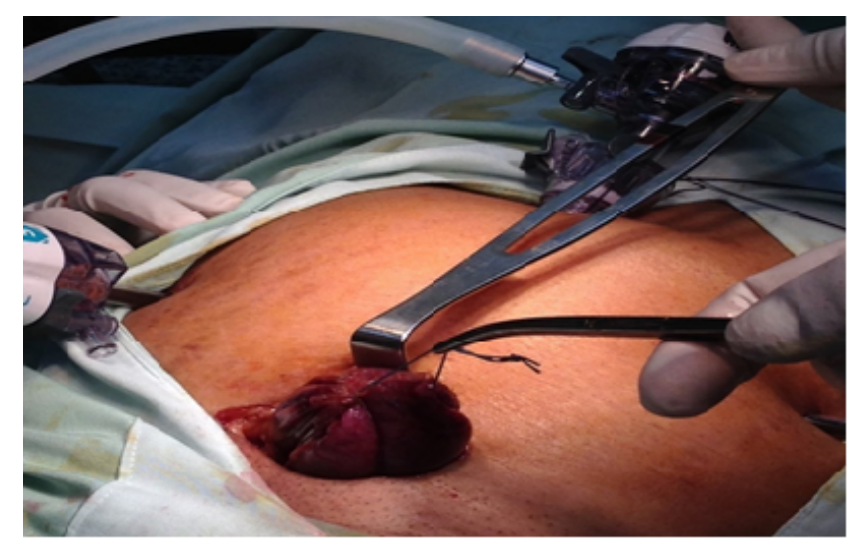

Figure 5. Intraoperative photograph showing the removal of gangrenous small bowel within a retrieval bag by an extended suprapubic port site incision.

Extra-corporal side to side anastomosis of small bowel was performed with an endo GI $45 \mathrm{~mm}$ stapler. There was no iatrogenic injury to the bowel, colon, the blood vessels or any other organs and the blood loss was minimal.

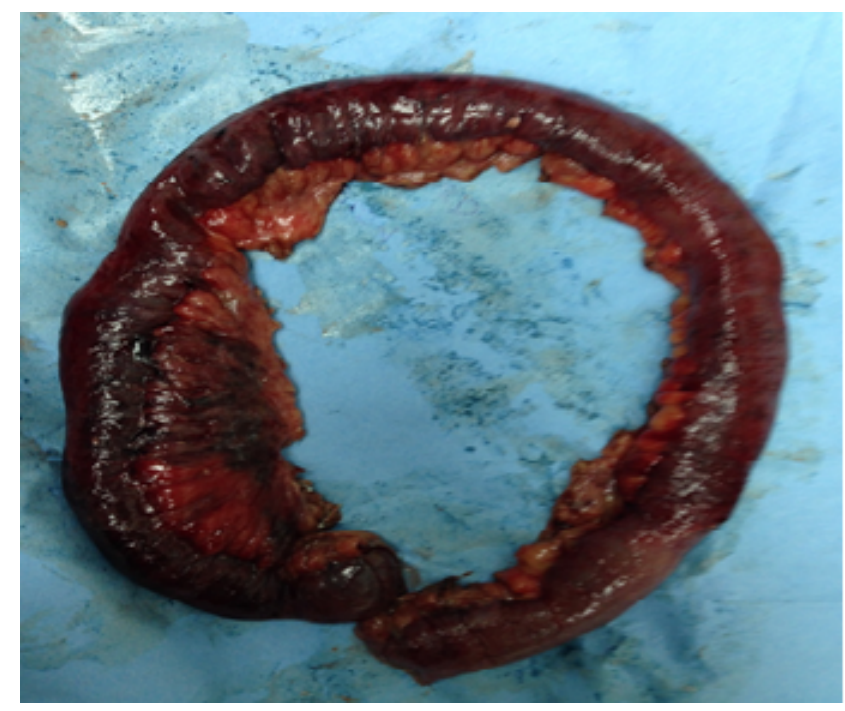

Figure 6. Resected segment of gangrenous small bowel

\section{Outcome and Follow up:}

The patient had a successful post-operative recovery with minimal analgesic requirement and was discharged home on the 4 th postoperative day (Figure 7). Histology confirmed the findings of a segment ischemic small bowel. At two year follow up, our patient was doing well with no further abdominal complaints (Figure 8).

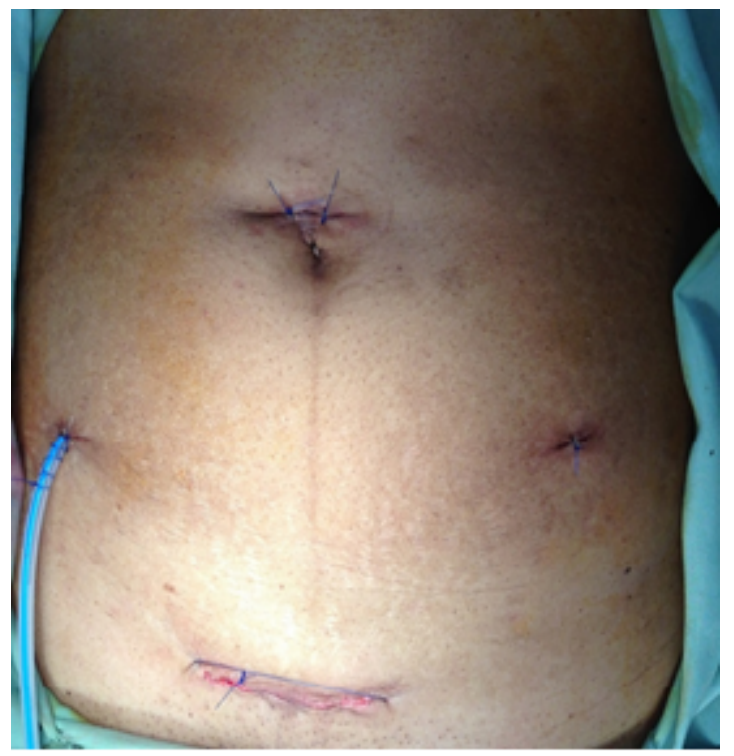

Figure 7. Intra operative photograph of the abdomen just before dressing

\section{DISCUSSION:}

Laparoscopic surgery has gained its popularity not only amongst the surgeons but also to the patients, because of its unique advantages: (1) less postoperative pain, (2) faster re- 


\section{Laparoscopic Resection of Gangrenous Small bowel with acute small bowel obstruction -}

World's First reported case

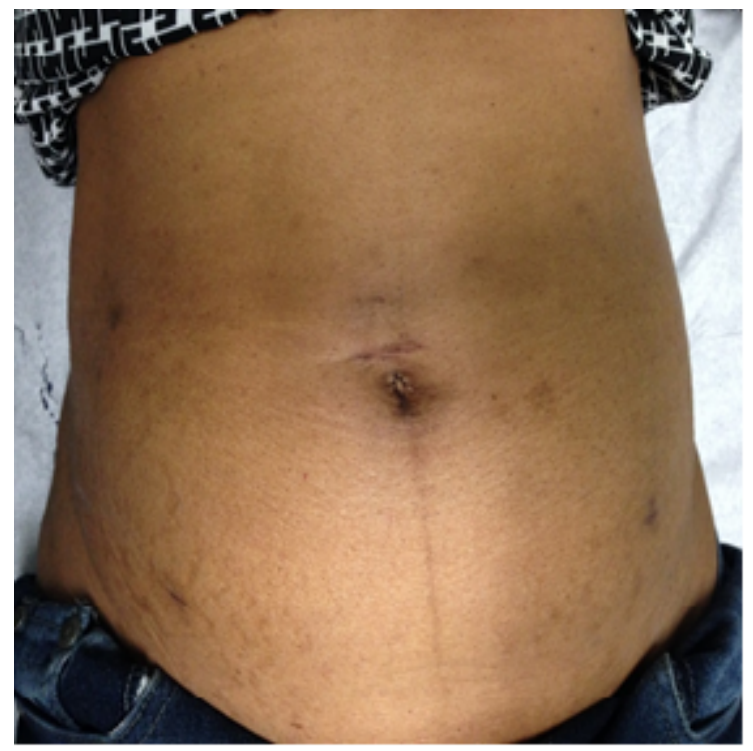

Figure 8. Photograph of the abdomenat 3 months follow up

turn of intestinal function, (3) shorter hospital stay, (4) reduced recovery time; allowing an earlier return to full activity, (5) decreased wound complications, and (6) decreased postoperative adhesion formation $[1,2]$.

Small bowel obstruction (SBO) is one of the most common causes of acute surgical admission. Mechanical small bowel obstruction, in particular the adhesion from previous surgeries, remains the predominant cause of SBO, accounting $60 \%$ to $70 \%$ of SBO $[3,4]$.

The cardinal symptoms of small bowel obstruction are abdominal pain, vomiting, distension and constipation [5]. The severity of these symptoms is directly related to the degree of vascular compromise rather than to the degree of intestinal obstruction [6].

Clinical history may suggest the possible cause of SBO. After an accurate physical examination and the evaluation of WBC, Lactate, Electrolytes, BUN and Creatinine; all patients being evaluated for small bowel obstruction should have plain abdominal films.

CT scan of the abdomen and pelvis should not be used routinely rather it should be preserved for the equivocal cases [7]. Prompt surgical intervention should be considered if there is CT scan evidence of any bowel ischemia as the longer waiting time before surgery is associated with increased risk of bowel resection [8].

The mainstays of management of adhesive SBO are initial trial of conservative treatment and if this fails, then proceed to either laparotomy or laparoscopy. However, for strangulating ASBO, open surgery is the preferred method of choice. On the contrary, the mainstay of management of non-adhesive SBO in a virgin abdomen is straight to surgery. Emergency laparotomy is indicated if there is sign and symptoms of acute abdomen with elevated WCC and CT scan evidence of free intraperitoneal fluid, mesenteric edema, lack of the "small bowel feces sign" and devascularized bowel [9]. However, this method of treatment should be reserved for selected cases only [10].

Diagnostic laparoscopy can play a vital role not only for the diagnosis but also for the treatment of selected cases of small bowel obstruction in a virgin abdomen. However, appropriate patient selection as well as the availability of a skilled laparoscopic surgeon is off paramount importance for a successful outcome [11].

Patients who are hemodynamically unstable and peritonitic with presence of free intra-abdominal air or gangrenous bowel are best managed with an open laparotomy. However, diagnostic laparoscopy can be a viable initial approach for patients without peritonitis and hemodynamic instability with a very low conversion rate of $8.7 \%$ [12]. Prompt surgical intervention should be performed as the success for early laparoscopic intervention for acute SBO is significantly higher when the duration of symptoms is shorter ( $24 \mathrm{~h}$ vs $48 \mathrm{~h}$ ) [13] and also when the cause of SBO is a single band like our patient [14].

Intraoperative patient selection during diagnostic laparoscopy is now being recommended by the Italian panel of experts on laparoscopic adhesiolysis [15]. The only absolute contra-indication mentioned by these groups is those related to pneumo-peritoneum. They suggested that each case should be judged based on its own merit depending on the laparoscopic skills of the surgeon [12].

During diagnostic laparoscopy atraumatic grasper should be used and the use of cautery should minimize to avoid any inadvertent bowel injury. Grasping of the dilated bowel should be avoided and proceed cautiously by holding the mesentery. The bowel should be followed up in a retrograde direction from the ileo-cecal valve towards the point of obstruction, thereby reducing the risk of handling the already dilated friable bowel.

Although until today, the literature has not yet documented a single case of emergency laparoscopic resection of gangrenous small bowel for SBO; the general principal of management of gangrenous bowel on laparoscopy is conversion to open resection [16] but this option should not be limited. It can be done laparoscopically in selected patients as shown in this case report. The necrotic small bowel needs to be handled carefully and grasping of the necrotic bowel should be avoided at any cost to avoid spillage and to prevent further contamination. The necrotic bowel also needs to be removed in a retriever bag. A very low threshold for open conversion should be maintained at all the time [17].

Despite its unique benefit, laparoscopic surgery however, is not always risk free. It is associated with intraoperative enterotomies ranging from $3 \%$ to $17.6 \%[18,19]$. One of the most dreaded complications of laparoscopic surgery is a missed enterotomy. However this can be minimized with meticulous handling of the mesentery rather than holding the friable bowel itself with an atraumatic grasper [20].

\section{CONCLUSION:}

Laparoscopic approach can be attempted in selected patients with first episode of SBO and/or anticipated single 
band obstruction. Similarly, gangrenous small bowel can also be resected laparoscopically in highly selected patients in the presence of an experienced laparoscopic surgeon. A complete SBO should no longer be considered an exclusion criterion for laparoscopic approach. This case report hereby provides the first evidence for and is able to break the traditional dogma of mandatory open laparotomy for the resection of gangrenous bowel on laparoscopy. However, a very low threshold for open conversion should always be maintained.

Learning Points:

1. Adhesive band (single) is a frequent cause of small bowel obstruction in virgin abdomen.

2. Surgery should not be delayed once there is clinical or radiological evidence of ischaemic bowel.

3. Laparotomy is an option but not always mandatory for resection of gangrenous bowel.

4. Laparoscopic resection of gangrenous small bowel is a feasible option in selected patients, in presence of a skilled laparoscopic surgeon.

\section{Patient perspective:}

I was very concerned initially for the large scar on my abdomen when the doctor told me that if the laparoscopic surgery fails they have to do an open laparotomy by a long midline incision. Thanks to the Lord that my doctors were able to do the surgery laparoscopically. I would like thank my doctors for their great effort and I am very happy with my overall outcome.

\section{Conflicts of interest:}

The authors declare no conflicts of interest.

\section{Author contribution:}

All authors have contributed significantly in designing and organizing to write manuscript, collecting data as well help in critical analyzing the manuscript. All authors have approved the final version of this manuscript

\section{Funding:}

No fund was received to publish this article.

\section{Consent:}

Patient consent was obtained.

\section{Guarantor:}

The corresponding author will accept the full responsibility for the work.

\section{REFERENCES}

[1] Nagle A, Ujiki M, Denham W, Murayama. K: Laparoscopic adhesiolysis for small bowel obstruction. Am J Surg;2004(187):464-470.

[2] Szomstein S, Lo Menzo E, Simpfendorfer C: Laparoscopic lysis of adhesions. World J Surg;2006(30):535-540.

[3] C MP. BJ: Postoperative adhesions: ten-year followup of 12,584 patients undergoing lower abdominal surgery. Dis Colon Rectum;2001(44):822-830.

[4] Ellis: The magnitude of adhesion related problems. Ann Chir Gynaecol1998;87:9-11.

[5] Zielinski MD, Bannon. MP: Current management of small bowel obstruction. Adv in Surg; 2011.
[6] Howard B, Steven W. Ozeran S: Factors predicting the recurrence of adhesive small-bowel obstruction. Am J Surg 1995;170(4):361-365.

[7] Obuz F, Terzi C, Sokmen S, Yilmaz E. Yildiz D, Fuzun M: The efficacy of helical CT in the diagnosis of small bowel obstruction. Eur J Radiol;2003(48):299-304.

[8] Leung $\mathrm{AM}, \mathrm{Vu} \mathrm{H}$ : Factors predicting need for and delay in surgery in small bowel obstruction. Am Surg;2012(78):403407.

[9] SaveriołSD, Coccolini F, Galati M, Nazareno. Smerieri et al. Bologna guidelines for diagnosis and management of adhesive small bowel obstruction (ASBO): 2013 update of the evidence-based guidelines from the world society of emergency surgery ASBO working group. World Journal of Emergency Surgery;8:42.

[10] Landercasper J, Cogbill TH, Merry WH. Stolee RT, Strutt PJ: Long-term outcome after hospitalization for small-bowel obstruction. Arch Surg; 1993.

[11] Ming-Zhe L, Lei L, Long-bin X, Wen-hui W. Yu-long H, Xinming S: Laparoscopic versus open adhesiolysis in patients with adhesive small bowel obstruction: a systematic review and meta-analysis. Am J Surg;2012(204).

[12] Vettoretto N, Carrara A, Corradi A, Vivo GD, Lazzaro L Ricciardelli L, et al. Tamborrino E, Zago M: Laparoscopic adhesiolysis: consensus conference guidelines. Colorectal diseases. The Association of Coloproctology of great Britain and Ireland;2012(14):208-2015.

[13] Levard H, Boudet MJ, Msika S, Molkhou JM, Hay JM, Laborde. Y: French association for surgical research. Laparoscopic treatment of acute small bowel obstruction: a multicentre retrospective;

[14] Navez B, Arimont JM, Guit P: Laparoscopic approach in acute small bowel obstruction. A review of 68 patients. Hepatogastroenterology; 1998.

[15] Agresta F, Ansaloni L, Baiocchi GL, Bergamini. C et al. Laparoscopic approach to acute abdomen from the Consensus Development Conference of the Società Italiana di Chirurgia Endoscopica e nuove tecnologie (SICE), Associazione Chirurghi Ospedalieri Italiani (ACOI), Società Italiana di Chirurgia (SIC), Società Italiana di Chirurgia d'Urgenza e del Trauma (SICUT), Società Italiana di Chirurgia nell'Ospedalità Privata (SICOP), and the European Association for Endoscopic Surgery (EAES). Surg Endosc;.

[16] Grafen FC, Neuhaus V, Schöb O. Turina M: Management of acute small bowel obstruction from intestinal adhesions: indications for laparoscopic surgery in a community teaching hospital. Langenbecks Arch Surg; 2010.

[17] Reschef A, Hull TL. Kiran RP: Risk of adhesive obstruction after colorectal surgery: the benefits of the minimally invasive approach may extend well beyond the perioperative period. Surg Endosc;2013:27-1717.

[18] Sato Y, Ido K, Kumagai M: Laparoscopic adhesiolysis for recurrent small bowel obstruction: long-term follow-up. Gastrointest Endosc;2001:54-476.

[19] Chosidow D, Johanet H. Montario T: Laparoscopy for acute small bowel obstruction secondary to adhesions. J Laparoendosc Adv Surg Tech;2000:10-155.

[20] Catena F. Di Saverio S, Ansaloni L: CHAPTER 7 Adhesive Small Bowel Obstruction. Updates in Surgery: The Role of Laparoscopy in Emergency Abdominal Surgery. Edited by: Mandalà V. 2012, Verlag Italia. Springer; 


\section{AUTHOR BIOGRAPHY}

Shariful Islam San Fernando Teaching Hospital, San Fernando, Trinidad and Tobago, Department of Clinical Surgical Science, University of the West Indies, St. Augustine, Trinidad and Tobago

Otis Payne MBBS, San Fernando Teaching Hospital, San Fernando, Trinidad and Tobago, Department of Clinical Surgical Science, University of the West Indies, St. Augustine, Trinidad and Tobago

Vinoo Bheem FRCS, San Fernando Teaching Hospital, San Fernando, Trinidad and Tobago

Patrick Harnarayan FRCS, FACS, San Fernando Teaching Hospital, San Fernando, Trinidad and Tobago, Department of Clinical Surgical Science, University of the West Indies, St. Augustine, Trinidad and Tobago

Dilip Dan FACS, San Fernando Teaching Hospital, San Fernando, Trinidad and Tobago, Department of Clinical Surgical Science, University of the West Indies, St. Augustine, Trinidad and Tobago 